\title{
Numerical Methods for a Class of Hybrid Weakly Singular Integro-Differential Equations
}

\author{
Shihchung Chiang \\ Department of Finance, Chung Hua University, Hsinchu, Taiwan \\ Email: Chiang@chu.edu.tw
}

How to cite this paper: Chiang, S. (2017) Numerical Methods for a Class of Hybrid Weakly Singular Integro-Differential Equations. Applied Mathematics, 8, 956-966. https://doi.org/10.4236/am.2017.87075

Received: May 25, 2017

Accepted: July 11, 2017

Published: July 14, 2017

Copyright $\odot 2017$ by author and Scientific Research Publishing Inc. This work is licensed under the Creative Commons Attribution International License (CC BY 4.0).

http://creativecommons.org/licenses/by/4.0/ (c) (i) Open Access

\begin{abstract}
This paper proposes numerical methods for solving hybrid weakly singular integro-differential equations of the second kind. The terms in these equations are in the following order: derivative term of a state, integro-differential term of a state with a weakly singular kernel, a state, integral term of a state with a smooth kernel, and force. The original class of weakly singular integro-differential equations of the first kind is derived from aeroelasticity mathematical models. Among the proposed methods, the method for solving linear cases is fully based on previously reported approximation scheme for equations of the first kind. For nonlinear cases, a revised method is proposed. Examples are presented to demonstrate the effectiveness of the proposed methods, and the results indicate that the proposed methods facilitate achieving satisfactory and accurate approximations.
\end{abstract}

\section{Keywords}

Hybrid, Weakly Singular, Integro-Differential Equations

\section{Introduction}

The original class of integro-differential equations is from an aeroelasticity problem, in which the mathematical model comprises eight integro-differential equations [1]. In this model, the most determinate equation is a scalar weakly singular integro-differential equation of the first kind. For the current study, a new equation comprising additional derivative terms and integro-differential terms with smooth kernel was used. Under an integrable assumption in previous studies, this new equation can be transformed into a Volterra integral equation of the second kind [2] [3] [4] [5] [6]. The remainder of this paper is organized as follows: Section 2 presents the equations. Section 3 presents the approach to the numerical methods from [7] for the linear cases and the revised version for the nonlinear cases. Section 4 presents the numerical results obtained by the me- 
thods in Section 4. Section 5 presents the summary.

\section{Problem Description}

Consider the class of hybrid weakly singular integro-differential equations

$$
\frac{\mathrm{d}}{\mathrm{d} t} x(t)+\frac{\mathrm{d}}{\mathrm{d} t} D x_{t}=x(t)+L x_{t}+f(t),
$$

the initial condition

$$
x(s)=\phi(s),-b \leq s \leq 0,
$$

where $b$ is a positive constant. The operators $D$ and $L$ are defined as follows:

$$
\begin{aligned}
& D x_{t}=\int_{-b}^{0} g(s) x_{t}(s) \mathrm{d} s \\
& L x_{t}=\int_{-b}^{0} c(s) x_{t}(s) \mathrm{d} s,
\end{aligned}
$$

where

$$
x_{t}(s)=x(t+s)
$$

The weighting kernel $g$ is integrable, positive, nondecreasing, and weakly singular at $s=0$. Kernel $c$ is smooth on $s$. The force $f(t)$ is assumed to be locally integrable for $t>0$. Although a more general kernel $g$ is suitable, in this study, emphasis was placed on the Abel type kernel and considers $g(s)=|s|^{-p}$ and $s \in[-b, 0]$ for $0<p<1$. A special value of $p=0.5$ corresponds to the original aeroelastic problem. The initial condition $\phi(s),-b \leq s \leq 0$ is in $L_{1, g}$ space, which is a weighted $L_{1}$ space with weight $g(\cdot)$. The initial value problems (1) and (2) can be expressed as

$$
x(t)+D x_{t}=D x_{0}+\phi(0)+\int_{0}^{t}\left(x(\tau)+\int_{-b}^{0} c(s) x(\tau+s) \mathrm{d} s+f(\tau)\right) \mathrm{d} \tau
$$

provided that the function

$$
t \rightarrow D x_{t}=\int_{-b}^{0} g(s) x(t+s) \mathrm{d} s
$$

is absolutely continuous and the function $g(\cdot) \phi(\cdot)$ belongs to $L_{1}[-b, 0]$. The corresponding weakly singular Volterra integral equation of the hybrid kind is

$$
\begin{aligned}
& x(t)+\int_{0}^{t}|s-t|^{-p} x(s) \mathrm{d} s \\
& =\phi(0)+\int_{-b}^{0}|s|^{-p} \phi(s) \mathrm{d} s+\int_{-b}^{0}\left(x(\tau)+\int_{-b}^{0} c(s) x(\tau+s) \mathrm{d} s+f(\tau)\right) \mathrm{d} \tau,
\end{aligned}
$$

for $0<t \leq b$.

The proposed algorithms use the separating variables method to directly solve Equations (1) and (2). Without loss of generality, assuming $b=1$, the equation is expressed as

$$
\frac{\mathrm{d}}{\mathrm{d} t} x(t)+\frac{\mathrm{d}}{\mathrm{d} t} \int_{-1}^{0}|s|^{-p} x(t+s) \mathrm{d} s=x(t)+\int_{-1}^{0} c(s) x(t+s) \mathrm{d} s+f(t),
$$

for $0<t \leq 1$, with initial data

$$
x(s)=\phi(s), s \leq 0,
$$


where $f(t), t>0$ is a locally integrable function. By the form of the state in the hybrid integro-differential Equation (8), we obtain the following result:

$$
\frac{\partial x(t+s)}{\partial s}=\frac{\partial x(t+s)}{\partial t},-1 \leq s \leq 0, t>0,
$$

and Equation (8) can be rewritten as

$$
\frac{\mathrm{d}}{\mathrm{d} t} x(t)+\int_{-1}^{0}|s|^{-p} \frac{\partial}{\partial s} x(t+s) \mathrm{d} s=x(t)+\int_{-1}^{0} c(s) x(t+s) \mathrm{d} s+f(t) .
$$

\section{Numerical Method}

\subsection{Linear Problems}

The proposed method entails discretizing Equation (1). The space mesh points (corresponding to the $s$ variable) are discretized as $-1=\tau_{n}<\tau_{n-1}<\cdots<\tau_{1}<\tau_{0}=0$, and a new variable $\xi$ is defined as follows:

$$
\xi(t, s)=x(t+s),-1 \leq s \leq 0,0<t .
$$

Equation (12) can then be reformulated as a first-order hyperbolic equation

$$
\frac{\partial}{\partial t} \xi(t, s)=\frac{\partial}{\partial s} \xi(t, s),-1 \leq s \leq 0
$$

with the condition

$$
\frac{\mathrm{d}}{\mathrm{d} t} \xi(t, 0)+\int_{-1}^{0}|s|^{-p} \frac{\partial}{\partial s} \xi(t, s) \mathrm{d} s=\xi(t, 0)+\int_{-1}^{0} c(s) \xi(t, s) \mathrm{d} s+f(t) .
$$

Next, assume that the solution to Equations (13) and (14) has the form

$$
\xi(t, s)=\sum_{i=0}^{n} \kappa_{i}(t) B_{i}(s)
$$

where the basis, $B_{i}(s), i=0, \cdots, n$ is given by

$$
B_{i}(s)=\left\{\begin{array}{llc}
\frac{1}{\tau_{i}-\tau_{i+1}}\left(s-\tau_{i+1}\right) & : & s \in\left[\tau_{i+1}, \tau_{i}\right] \\
\frac{1}{\tau_{i-1}-\tau_{i 1}}\left(\tau_{i-1}-s\right) & : & s \in\left[\tau_{i}, \tau_{i-1}\right], \\
0 & : & \text { otherwise }
\end{array}\right.
$$

where $B_{i}(s), i=0, \cdots, n$ is a piecewise linear function. After substituting the special form of $\xi$ expressed in Equation (15) into Equations (13) and (14), the governing equations for $\kappa_{j}(t), j=0, \cdots, n$ can be expressed as follows:

$$
\begin{gathered}
\frac{\mathrm{d}}{\mathrm{d} t} \kappa_{i}(t)=\frac{1}{\delta_{i}}\left(\kappa_{i-1}(t)-\kappa_{i}(t)\right), i=1, \cdots, n, \\
\frac{\mathrm{d}}{\mathrm{d} t} \sum_{i=0}^{n} \kappa_{i}(t) B_{i}(0)+\int_{-1}^{0}|s|^{-p} \sum_{i=0}^{n} \kappa_{i}(t) \frac{\mathrm{d}}{\mathrm{d} s} B_{i}(s) \mathrm{d} s \\
=\kappa_{i}(t) B_{i}(0)+\int_{-1}^{0} c(s) \sum_{i=0}^{n} \kappa_{i}(t) B_{i}(s) \mathrm{d} s+f(t),
\end{gathered}
$$

where $\delta_{i}=\tau_{i-1}-\tau_{i}>0$, for $i=1, \cdots, n$. By defining constants $c_{i}$ and $d_{i}$, for 
$i=0, \cdots, n$, and applying the property of $B_{i}(s)$, Equation (17) can be written as

$$
\frac{\mathrm{d}}{\mathrm{d} t} \kappa_{0}(t)+\sum_{i=0}^{n} c_{i} \kappa_{i}(t)=\kappa_{0}(t)+\sum_{i=0}^{n} d_{i} \kappa_{i}(t)+f(t) .
$$

Note that Equations (16) and (18) can form a system of first order ordinary differential equations. For time $t$, the discretization contains $T^{0}, T^{1}, \cdots, T^{m}$, for $0=T^{0}<T^{1}<\cdots<T^{m}=1$. Define $\Delta^{k}=T^{k+1}-T^{k}$, for $k=0, \cdots, m-1$. Assume $\alpha_{i}^{k}=\kappa_{i}\left(T^{k}\right)$, for $i=0, \cdots, n$, and $k=0, \cdots, m$. With first term of Equation (18) replaced by the first order finite difference, Equations (16) and (18) can now be expressed as follows:

$$
\frac{1}{\Delta^{k}}\left(\alpha_{0}^{k+1}-\alpha_{0}^{k}\right)+\sum_{i=0}^{n} c_{i} \alpha_{i}^{k+1}=\alpha_{0}^{k+1}+\sum_{i=0}^{n} d_{i} \alpha_{i}^{k+1}+f\left(T^{k+1}\right),
$$

for $k=0, \cdots, m-1$. Furthermore, assume a uniform mesh for both space and time variables, the mesh points are $\tau_{i}, i=0, \cdots, n$, and $T^{k}, k=0, \cdots, m$. Specifically, $\tau_{i}=-\frac{i}{n}$ and $T^{k}=\frac{k}{m}$ for some positive integers $n$ and $m$. The corresponding differences are defined as $\Delta^{k}=T^{k+1}-T^{k}, \quad k=0, \cdots, m-1$, for the time variable, and $\delta_{i}=\tau_{i-1}-\tau_{i}, \quad i=1, \cdots, n$, for the space variable. Thus, $\Delta^{k}=\frac{1}{m}$ and $\delta_{i}=\frac{1}{n}$ for $k=0, \cdots, m-1$ and $i=1, \cdots, n$. Setting $m=n$ leads to the relation $\Delta^{k}=\delta_{i}=\frac{1}{n}$ for $k=0, \cdots, m-1$ and $i=1, \cdots, n$ and Equations (19) and (18) lead to the following system:

$$
\alpha_{i}^{k+1}=\alpha_{i-1}^{k},
$$

and

$$
\begin{aligned}
& \frac{\alpha_{0}^{k+1}-\alpha_{0}^{k}}{\delta_{k+1}}+\alpha_{0}^{k+1}\left(c_{0}-d_{0}-1\right)+\alpha_{1}^{k+1}\left(c_{1}-d_{1}\right) \\
& +\cdots+\alpha_{n}^{k+1}\left(c_{n}-d_{n}\right)=f\left(T^{k+1}\right),
\end{aligned}
$$

for $k=0,1, \cdots, n-1$. After defining the corresponding constants $e_{0}, e_{1}, \cdots, e_{n}$, Equation (21) can be simplified as follows:

$$
\alpha_{0}^{k+1} e_{0}+\alpha_{1}^{k+1} e_{1}+\cdots+\alpha_{i}^{k+1} e_{k+1}+\cdots+\alpha_{n}^{k+1} e_{n}=f\left(T^{k+1}\right),
$$

for $k=0,1, \cdots, n-1$. The connection of the solution $x(t)$ and $\alpha$ 's is as follows: Because $\xi(t, s)=x(t+s)$, for $-1 \leq s \leq 0$ and $t>0$, and $\xi(t, s)=\sum_{i=0}^{n} \kappa_{i}(t) B_{i}(s) . x(\bar{t})$ can be obtained for $1 \geq \bar{t}>0$ in the following case:

$$
x\left(T^{j}\right)=\sum_{l=0}^{n} \kappa_{l}\left(T^{j}\right) B_{l}(0)=\kappa_{0}\left(T^{j}\right)=\alpha_{0}^{j}, \text { for } j=1, \cdots, n .
$$

Equations above with the initial condition can be set up as $[A][x]=[b]$, where the vector $[x]$ comprises the unknowns $\alpha_{0}^{j}, j=1, \cdots, n$. The structure of ma- 
$\operatorname{trix}[A]$ is

$$
\left[\begin{array}{cccccc}
e_{0} & 0 & \cdots & 0 & \cdots & 0 \\
e_{1} & e_{0} & 0 & \cdots & \cdots & 0 \\
\vdots & \ddots & \vdots & \vdots & \ddots & \vdots \\
e_{n-1} & e_{n-2} & \cdots & \cdots & \cdots & e_{0}
\end{array}\right]_{n \times n}
$$

and that of vector $[b]$ is

$$
\left[\begin{array}{c}
f\left(T^{1}\right)-\alpha_{0}^{0} e_{1}-\alpha_{1}^{0} e_{2}-\cdots-\alpha_{n-1}^{0} e_{n} \\
f\left(T^{2}\right)-\alpha_{0}^{0} e_{2}-\alpha_{1}^{0} e_{3}-\cdots-\alpha_{n-2}^{0} e_{n} \\
\vdots \\
f\left(T^{n}\right)-\alpha_{0}^{0} e_{n}
\end{array}\right]_{n \times 1}
$$

\subsection{Nonlinear Problems}

The second proposed method contains part of the first method. By assuming $x(t+s)=\xi(t, s)$, then the property of Equation (13):

$$
\frac{\partial}{\partial t} \xi(t, s)=\frac{\partial}{\partial s} \xi(t, s)
$$

for $-1 \leq s \leq 0$ still holds. The discretized Equation (1) follows the study [7]:

$$
\begin{aligned}
& \frac{\mathrm{d}}{\mathrm{d} t} x(t)+\sum_{j=0}^{n / 2-1} \int_{\tau_{2 j+2}}^{\tau_{2 j}}|s|^{-p} \frac{x\left(t+\tau_{2 j}\right)-x\left(t+\tau_{2 j+2}\right)}{2 \Delta s} \mathrm{~d} s \\
& =x(t)+\sum_{j=0}^{n / 2-1} \int_{\tau_{2 j+2}}^{\tau_{2 j}} c(s) x\left(t+\tau_{2 j+1}\right) \mathrm{d} s+f(t),
\end{aligned}
$$

for $n$ is even. $\Delta s=\tau_{j+1}-\tau_{j}$, for $j=0,1, \cdots, n-1$ is constant for uniform mesh. By applying the property of $\tau_{i}, i=0,1, \cdots, n$, Equation (24) can be written as

$$
\begin{aligned}
& \frac{\mathrm{d}}{\mathrm{d} t} \xi(t, 0)+\xi(t, 0)\left[\frac{\left(-\tau_{2}\right)^{1-p}}{2 \Delta s(1-p)}-1\right] \\
& =\xi\left(t, \tau_{2}\right)\left[\frac{\left(-\tau_{2}\right)^{1-p}}{2 \Delta s(1-p)}\right] \\
& \quad+\frac{1}{2 \Delta s(1-p)} \sum_{j=0}^{n / 2-1}\left[\left(-\tau_{2 j}\right)^{1-p}-\left(-\tau_{2 j+2}\right)^{1-p}\right] \\
& \quad \times\left[\xi\left(t, \tau_{2 j}\right)-\xi\left(t, \tau_{2 j+2}\right)\right]+2 \Delta s \sum_{j=0}^{n / 2-1} c\left(\tau_{2 j+1}\right) \xi\left(t, \tau_{2 j+1}\right)+f(t) .
\end{aligned}
$$

Next, assume that

$$
\xi(t, s)=\sum_{i=0}^{n} \kappa_{i}(t) B_{i}(s)
$$

where the basis, $B_{i}(s), i=0,1, \cdots, n$ are the same as above.

$$
\text { 3.2.1. } \mathrm{c}(\mathrm{s})=1
$$

Equation (25) becomes 


$$
\begin{aligned}
& \frac{\mathrm{d}}{\mathrm{d} t} \kappa_{0}(t)+\kappa_{0}(t)\left[\frac{\left(-\tau_{2}\right)^{1-p}}{2 \Delta s(1-p)}-1\right] \\
& =\kappa_{2}(t)\left[\frac{\left(-\tau_{2}\right)^{1-p}}{2 \Delta s(1-p)}\right] \\
& \quad+\frac{1}{2 \Delta s(1-p)} \sum_{j=0}^{n / 2-1}\left[\left(-\tau_{2 j}\right)^{1-p}-\left(-\tau_{2 j+2}\right)^{1-p}\right] \\
& \quad \times\left[\kappa_{2 j}(t)-\kappa_{2 j+2}(t)\right]+2 \Delta s \sum_{j=0}^{n / 2-1} \kappa_{2 j+1}(t)+f(t) .
\end{aligned}
$$

Setting $t=T^{1}$, and assuming $\frac{\mathrm{d}}{\mathrm{d} t} \kappa_{0}\left(T^{1}\right)=\frac{\kappa_{0}\left(T^{2}\right)-\kappa_{0}\left(T^{0}\right)}{2 \Delta s}$, then

$$
\begin{aligned}
& \frac{\kappa_{0}\left(T^{2}\right)}{2 \Delta s}+\kappa_{0}\left(T^{1}\right)\left[\frac{\left(-\tau_{2}\right)^{1-p}}{2 \Delta s(1-p)}-1\right] \\
& =\frac{\phi(0)}{2 \Delta s}+\phi\left(\tau_{1}\right)\left[\frac{\left(-\tau_{2}\right)^{1-p}}{2 \Delta s(1-p)}\right] \\
& \quad+\frac{1}{2 \Delta s(1-p)} \sum_{j=0}^{n / 2-1}\left[\left(-\tau_{2 j}\right)^{1-p}-\left(-\tau_{2 j+2}\right)^{1-p}\right] \\
& \quad \times\left[\phi\left(\tau_{2 j-1}\right)-\phi\left(\tau_{2 j+1}\right)\right]+2 \Delta s \sum_{j=0}^{n / 2-1} \phi\left(\tau_{2 j}\right)+f\left(T^{1}\right) .
\end{aligned}
$$

Similarly, setting $t=T^{2}$, then

$$
\begin{aligned}
& \frac{\kappa_{0}\left(T^{3}\right)}{2 \Delta s}+\kappa_{0}\left(T^{2}\right)\left[\frac{\left(-\tau_{2}\right)^{1-p}}{2 \Delta s(1-p)}-1\right]+\kappa_{0}\left(T^{1}\right)\left[-\frac{1}{2 \Delta s}-2 \Delta s\right] \\
& =\phi(0)\left[\frac{\left(-\tau_{2}\right)^{1-p}}{2 \Delta s(1-p)}\right]+\frac{1}{2 \Delta s(1-p)} \sum_{j=1}^{n / 2-1}\left[\left(-\tau_{2 j}\right)^{1-p}-\left(-\tau_{2 j+2}\right)^{1-p}\right] \\
& \times\left[\phi\left(\tau_{2 j-2}\right)-\phi\left(\tau_{2 j}\right)\right]+2 \Delta s \sum_{j=1}^{n / 2-1} \phi\left(\tau_{2 j-1}\right)+f\left(T^{2}\right) .
\end{aligned}
$$

For $3 \leq i \leq n / 2-1$ and $t=T^{2 i-1}$,

$$
\begin{aligned}
& \frac{\kappa_{0}\left(T^{2 i}\right)}{2 \Delta s}+\kappa_{0}\left(T^{2 i-1}\right)\left[\frac{\left(-\tau_{2}\right)^{1-p}}{2 \Delta s(1-p)}-1\right]+\kappa_{0}\left(T^{2 i-2}\right)\left[-\frac{1}{2 \Delta s}-2 \Delta s\right]+\cdots \\
& +\kappa_{0}\left(T^{1}\right) \frac{1}{2 \Delta s(1-p)}\left[\left(-\tau_{2 i-4}\right)^{1-p}-2\left(-\tau_{2 i-2}\right)^{1-p}+\left(-\tau_{2 i}\right)^{1-p}\right] \\
& =\phi\left(\tau_{1}\right)\left[\frac{-1}{2 \Delta s(1-p)}\right]\left[\left(-\tau_{2 i-2}\right)^{1-p}-\left(-\tau_{2 i}\right)^{1-p}\right] \\
& +\frac{1}{2 \Delta s(1-p)} \sum_{j=i}^{n / 2-1}\left[\left(-\tau_{2 j}\right)^{1-p}-\left(-\tau_{2 j+2}\right)^{1-p}\right]\left[\phi\left(\tau_{2 j-2 i+1}\right)-\phi\left(\tau_{2 j-2 i+3}\right)\right] \\
& +2 \Delta s \sum_{j=i-1}^{n / 2-1} \phi\left(\tau_{2 j-2 i+2}\right)+f\left(T^{2 i-1}\right) .
\end{aligned}
$$

For $t=T^{2 i}$, 


$$
\begin{aligned}
& \frac{\kappa_{0}\left(T^{2 i+1}\right)}{2 \Delta s}+\kappa_{0}\left(T^{2 i}\right)\left[\frac{\left(-\tau_{2}\right)^{1-p}}{2 \Delta s(1-p)}-1\right]+\kappa_{0}\left(T^{2 i-1}\right)\left[-\frac{1}{2 \Delta s}-2 \Delta s\right]+\cdots+\kappa_{0}\left(T^{1}\right)[-2 \Delta s] \\
& =\phi(0)\left[\frac{-1}{2 \Delta s(1-p)}\right]\left[\left(-\tau_{2 i-2}\right)^{1-p}-\left(-\tau_{2 i}\right)^{1-p}\right] \\
& +\frac{1}{2 \Delta s(1-p)} \sum_{j=i}^{n / 2-1}\left[\left(-\tau_{2 j}\right)^{1-p}-\left(-\tau_{2 j+2}\right)^{1-p}\right]\left[\phi\left(\tau_{2 j-2 i}\right)-\phi\left(\tau_{2 j-2 i+2}\right)\right] \\
& +2 \Delta s \sum_{j=i}^{n / 2-1} \phi\left(\tau_{2 j-2 i+1}\right)+f\left(T^{2 i}\right) \text {, } \\
& \text { then, for } t=T^{n-1} \text {, } \\
& \frac{\kappa_{0}\left(T^{n}\right)}{2 \Delta s}+\kappa_{0}\left(T^{n-1}\right)\left[\frac{\left(-\tau_{2}\right)^{1-p}}{2 \Delta s(1-p)}-1\right]+\kappa_{0}\left(T^{n-2}\right)\left[-\frac{1}{2 \Delta s}-2 \Delta s\right]+\cdots \\
& +\kappa_{0}\left(T^{1}\right) \frac{1}{2 \Delta s(1-p)}\left[\left(-\tau_{n-4}\right)^{1-p}-2\left(-\tau_{n-2}\right)^{1-p}+\left(-\tau_{n}\right)^{1-p}\right] \\
& =\phi\left(\tau_{1}\right)\left[\frac{-1}{2 \Delta s(1-p)}\right]\left[\left(-\tau_{n-2}\right)^{1-p}-\left(-\tau_{n}\right)^{1-p}\right] \\
& +\frac{1}{2 \Delta s(1-p)} \sum_{j=i}^{n / 2-1}\left[\left(-\tau_{2 j}\right)^{1-p}-\left(-\tau_{2 j+2}\right)^{1-p}\right]\left[\phi\left(\tau_{2 j-2 i+1}\right)-\phi\left(\tau_{2 j-2 i+3}\right)\right]+2 \Delta s \phi(0)+f\left(T^{n-1}\right), \\
& \text { and for } t=T^{n} \text {, } \\
& \kappa_{0}\left(T^{n}\right)\left[\frac{1}{2 \Delta s}+\frac{\left(-\tau_{2}\right)^{1-p}}{2 \Delta s(1-p)}-1\right]+\kappa_{0}\left(T^{1}\right)[-2 \Delta s] \\
& +\kappa_{0}\left(T^{n-2}\right)\left[\frac{-1}{2 \Delta s}-\frac{2\left(-\tau_{2}\right)^{1-p}+\left(-\tau_{4}\right)^{1-p}}{2 \Delta s(1-p)}\right]+\cdots+\kappa_{0}\left(T^{1}\right)[-2 \Delta s] \\
& =\phi(0)\left[\frac{-1}{2 \Delta s(1-p)}\right]\left[\left(-\tau_{n-2}\right)^{1-p}-\left(-\tau_{n}\right)^{1-p}\right]+f\left(T^{n}\right) .
\end{aligned}
$$

By collecting Equations (28), (29), (30), (31), (32), (33) and assuming $\alpha_{0}^{k}=\kappa_{0}\left(T^{k}\right)$, for $k=0,1, \cdots, n$, the system $[A][x]=[b]$ is constructed, where

$$
[A]=\left[\begin{array}{ccccccc}
\frac{\left(-\tau_{2}\right)^{1-p}}{2 \Delta s(1-p)}-1 & \frac{1}{2 \Delta s} & 0 & \cdots & 0 & \cdots & 0 \\
* & * & * & 0 & \cdots & \cdots & 0 \\
\vdots & \ddots & \vdots & \vdots & \ddots & \vdots & \vdots \\
* & * & * & \cdots & \cdots & \cdots & \cdots
\end{array}\right]_{n \times n}
$$

and that of vector $[b]$ is

$$
[b]=\left[\begin{array}{c}
f\left(T^{1}\right)+\phi(0) \frac{1}{2 \Delta s}+\cdots+2 \Delta s \sum_{j=0}^{n / 2-1} \phi\left(\tau_{2 j}\right) \\
f\left(T^{2}\right)+\phi(0) \frac{\left(-\tau_{2}\right)^{1-p}}{2 \Delta s(1-p)}+\cdots+2 \Delta s \sum_{j=1}^{n / 2-1} \phi\left(\tau_{2 j-1}\right) \\
\vdots \\
f\left(T^{n}\right)-\phi(0) \frac{\left(-\tau_{n-2}\right)^{1-p}-\left(-\tau_{n}\right)^{1-p}}{2 \Delta s(1-p)}
\end{array}\right]_{n \times 1}
$$


3.2.2. $\mathrm{c}(\mathrm{s})=\mathrm{s}$

In this case, Equation (25) becomes

$$
\begin{aligned}
& \frac{\mathrm{d}}{\mathrm{d} t} \kappa_{0}(t)+\kappa_{0}(t)\left[\frac{\left(-\tau_{2}\right)^{1-p}}{2 \Delta s(1-p)}-1\right] \\
& =\kappa_{2}(t)\left[\frac{\left(-\tau_{2}\right)^{1-p}}{2 \Delta s(1-p)}\right] \\
& \quad+\frac{1}{2 \Delta s(1-p)} \sum_{j=0}^{n / 2-1}\left[\left(-\tau_{2 j}\right)^{1-p}-\left(-\tau_{2 j+2}\right)^{1-p}\right] \\
& \quad \times\left[\kappa_{2 j}(t)-\kappa_{2 j+2}(t)\right]+2 \Delta s \sum_{j=0}^{n / 2-1} \tau_{2 j+1} \kappa_{2 j+1}(t)+f(t),
\end{aligned}
$$

Setting $t=T^{1}$, and assuming $\frac{\mathrm{d}}{\mathrm{d} t} \kappa_{0}\left(T^{1}\right)=\frac{\kappa_{0}\left(T^{2}\right)-\kappa_{0}\left(T^{0}\right)}{2 \Delta s}$, then

$$
\begin{aligned}
& \frac{\kappa_{0}\left(T^{2}\right)}{2 \Delta s}+\kappa_{0}\left(T^{1}\right)\left[\frac{\left(-\tau_{2}\right)^{1-p}}{2 \Delta s(1-p)}-1\right] \\
& =\frac{\phi(0)}{2 \Delta s}+\phi\left(\tau_{1}\right)\left[\frac{\left(-\tau_{2}\right)^{1-p}}{2 \Delta s(1-p)}\right] \\
& \quad+\frac{1}{2 \Delta s(1-p)} \sum_{j=0}^{n / 2-1}\left[\left(-\tau_{2 j}\right)^{1-p}-\left(-\tau_{2 j+2}\right)^{1-p}\right] \\
& \quad \times\left[\phi\left(\tau_{2 j-1}\right)-\phi\left(\tau_{2 j+1}\right)\right]+2 \Delta s \sum_{j=0}^{n / 2-1} \tau_{2 j+1} \phi\left(\tau_{2 j}\right)+f\left(T^{1}\right) .
\end{aligned}
$$

Similarly, setting $t=T^{2}$, then

$$
\begin{aligned}
& \frac{\kappa_{0}\left(T^{3}\right)}{2 \Delta s}+\kappa_{0}\left(T^{2}\right)\left[\frac{\left(-\tau_{2}\right)^{1-p}}{2 \Delta s(1-p)}-1\right]+\kappa_{0}\left(T^{1}\right)\left[-\frac{1}{2 \Delta s}-2 \Delta s\right] \\
& =\phi(0)\left[\frac{\left(-\tau_{2}\right)^{1-p}}{2 \Delta s(1-p)}\right]+\frac{1}{2 \Delta s(1-p)} \sum_{j=1}^{n / 2-1}\left[\left(-\tau_{2 j}\right)^{1-p}-\left(-\tau_{2 j+2}\right)^{1-p}\right] \\
& \quad \times\left[\phi\left(\tau_{2 j-2}\right)-\phi\left(\tau_{2 j}\right)\right]+2 \Delta s \sum_{j=1}^{n / 2-1} \tau_{2 j+1} \phi\left(\tau_{2 j-1}\right)+f\left(T^{2}\right) .
\end{aligned}
$$

For $3 \leq i \leq n / 2-1$ and $t=T^{2 i-1}$,

$$
\begin{aligned}
& \frac{\kappa_{0}\left(T^{2 i}\right)}{2 \Delta s}+\kappa_{0}\left(T^{2 i-1}\right)\left[\frac{\left(-\tau_{2}\right)^{1-p}}{2 \Delta s(1-p)}-1\right]+\kappa_{0}\left(T^{2 i-2}\right)\left[-\frac{1}{2 \Delta s}-2 \Delta s \tau_{1}\right]+\cdots \\
& +\kappa_{0}\left(T^{1}\right) \frac{1}{2 \Delta s(1-p)}\left[\left(-\tau_{2 i-4}\right)^{1-p}-2\left(-\tau_{2 i-2}\right)^{1-p}+\left(-\tau_{2 i}\right)^{1-p}\right] \\
& =\phi\left(\tau_{1}\right)\left[\frac{-1}{2 \Delta s(1-p)}\right]\left[\left(-\tau_{2 i-2}\right)^{1-p}-\left(-\tau_{2 i}\right)^{1-p}\right] \\
& +\frac{1}{2 \Delta s(1-p)} \sum_{j=i}^{n / 2-1}\left[\left(-\tau_{2 j}\right)^{1-p}-\left(-\tau_{2 j+2}\right)^{1-p}\right]\left[\phi\left(\tau_{2 j-2 i+1}\right)-\phi\left(\tau_{2 j-2 i+3}\right)\right] \\
& +2 \Delta s \sum_{j=i-1}^{n / 2-1} \tau_{2 j+1} \phi\left(\tau_{2 j-2 i+2}\right)+f\left(T^{2 i-1}\right) .
\end{aligned}
$$

For $t=T^{2 i}$, 


$$
\begin{gathered}
\frac{\kappa_{0}\left(T^{2 i+1}\right)}{2 \Delta s}+\kappa_{0}\left(T^{2 i}\right)\left[\frac{\left(-\tau_{2}\right)^{1-p}}{2 \Delta s(1-p)}-1\right]+\kappa_{0}\left(T^{2 i-1}\right)\left[-\frac{1}{2 \Delta s}-2 \Delta s \tau_{1}\right]+\cdots+\kappa_{0}\left(T^{1}\right)\left[-2 \Delta s \tau_{2 i-1}\right] \\
=\phi(0)\left[\frac{-1}{2 \Delta s(1-p)}\right]\left[\left(-\tau_{2 i-2}\right)^{1-p}-\left(-\tau_{2 i}\right)^{1-p}\right] \\
+\frac{1}{2 \Delta s(1-p)} \sum_{j=i}^{n / 2-1}\left[\left(-\tau_{2 j}\right)^{1-p}-\left(-\tau_{2 j+2}\right)^{1-p}\right]\left[\phi\left(\tau_{2 j-2 i}\right)-\phi\left(\tau_{2 j-2 i+2}\right)\right] \\
+2 \Delta s \sum_{j=i}^{n / 2-1} \tau_{2 j+1} \phi\left(\tau_{2 j-2 i+1}\right)+f\left(T^{2 i}\right), \\
\text { then, for } t=T^{n-1}, \\
\frac{\kappa_{0}\left(T^{n}\right)}{2 \Delta s}+\kappa_{0}\left(T^{n-1}\right)\left[\frac{\left(-\tau_{2}\right)^{1-p}}{2 \Delta s(1-p)}-1\right]+\kappa_{0}\left(T^{n-2}\right)\left[-\frac{1}{2 \Delta s}-2 \Delta s \tau_{1}\right]+\cdots \\
+\kappa_{0}\left(T^{1}\right) \frac{1}{2 \Delta s(1-p)}\left[\left(-\tau_{n-4}\right)^{1-p}-2\left(-\tau_{n-2}\right)^{1-p}+\left(-\tau_{n}\right)^{1-p}\right] \\
=\phi\left(\tau_{1}\right)\left[\frac{-1}{2 \Delta s(1-p)}\right]\left[\left(-\tau_{n-2}\right)^{1-p}-\left(-\tau_{n}\right)^{1-p}\right]+2 \Delta s \tau_{n-1} \phi(0)+f\left(T^{n-1}\right)
\end{gathered}
$$

and for $t=T^{n}$,

$$
\begin{aligned}
& \kappa_{0}\left(T^{n}\right)\left[\frac{1}{2 \Delta s}+\frac{\left(-\tau_{2}\right)^{1-p}}{2 \Delta s(1-p)}-1\right]+\kappa_{0}\left(T^{1}\right)\left[-2 \Delta s \tau_{1}\right] \\
& +\kappa_{0}\left(T^{n-2}\right)\left[\frac{-1}{2 \Delta s}-\frac{2\left(-\tau_{2}\right)^{1-p}+\left(-\tau_{4}\right)^{1-p}}{2 \Delta s(1-p)}\right]+\cdots+\kappa_{0}\left(T^{1}\right)\left[-2 \Delta s \tau_{n-1}\right] \\
& =\phi(0)\left[\frac{-1}{2 \Delta s(1-p)}\right]\left[\left(-\tau_{n-2}\right)^{1-p}-\left(-\tau_{n}\right)^{1-p}\right]+f\left(T^{n}\right) .
\end{aligned}
$$

By collecting Equations (35), (36), (37), (38), (39), (40) and assuming $\alpha_{0}^{k}=\kappa_{0}\left(T^{k}\right)$, for $k=0,1, \cdots, n$, the system $[A][x]=[b]$ is constructed, where

$$
[A]=\left[\begin{array}{ccccccc}
\frac{\left(-\tau_{2}\right)^{1-p}}{2 \Delta s(1-p)}-1 & \frac{1}{2 \Delta s} & 0 & \cdots & 0 & \cdots & 0 \\
* & * & * & 0 & \cdots & \cdots & 0 \\
\vdots & \ddots & \vdots & \vdots & \ddots & \vdots & \vdots \\
* & * & * & \cdots & \cdots & \cdots & \cdots
\end{array}\right]_{n \times n}
$$

and that of vector $[b]$ is

$$
[b]=\left[\begin{array}{c}
f\left(T^{1}\right)+\phi(0) \frac{1}{2 \Delta s}+\cdots+2 \Delta s \sum_{j=0}^{n / 2-1} \tau_{2 j+1} \phi\left(\tau_{2 j}\right) \\
f\left(T^{2}\right)+\phi(0) \frac{\left(-\tau_{2}\right)^{1-p}}{2 \Delta s(1-p)}+\cdots+2 \Delta s \sum_{j=1}^{n / 2-1} \tau_{2 j+1} \phi\left(\tau_{2 j-1}\right) \\
\vdots \\
f\left(T^{n}\right)-\phi(0) \frac{\left(-\tau_{n-2}\right)^{1-p}-\left(-\tau_{n}\right)^{1-p}}{2 \Delta s(1-p)}
\end{array}\right]_{n \times 1}
$$




\section{Numerical Examples}

A desktop computer (Intel Pentium 4 microprocessor, $2.80 \mathrm{GHz} \mathrm{CPU}$, and 224 MB RAM) was used for testing the examples.

Example 1. $c(s)=1, \phi(s)=s, f(t)=3.5-2 t, x(t)=t$.

\begin{tabular}{ccc}
\hline method & Max error & $n$ \\
\hline direct & $5.4769 \mathrm{e}-13$ & 100 \\
direct & $2.7453 \mathrm{e}-10$ & 1000 \\
revised & $6.1062 \mathrm{e}-15$ & 100 \\
revised & $6.2506 \mathrm{e}-14$ & 1000 \\
\hline
\end{tabular}

Example 2. $c(s)=1, \phi(s)=0, f(t)=1+2 t^{0.5}-t-t^{2}, x(t)=t$.

\begin{tabular}{ccc}
\hline method & Max error & $n$ \\
\hline direct & $4.0992 \mathrm{e}-13$ & 100 \\
direct & $2.6994 \mathrm{e}-10$ & 1000 \\
revised & 0.0014 & 100 \\
revised & $4.6559 \mathrm{e}-5$ & 1000 \\
\hline
\end{tabular}

Example 3. $c(s)=1, \phi(s)=s^{2}, f(t)=-5 / 3+7 t-2 t^{2}, x(t)=t^{2}$.

\begin{tabular}{ccc}
\hline method & Max error & $n$ \\
\hline direct & 4.3806 & 100 \\
direct & 4.3801 & 1000 \\
revised & 0.0231 & 100 \\
revised & 0.0026 & 1000 \\
\hline
\end{tabular}

Example 4. $c(s)=s, \phi(s)=s, f(t)=8 / 3-t / 2, x(t)=t$.

\begin{tabular}{ccc}
\hline method & Max error & $n$ \\
\hline direct & $6.4217 \mathrm{e}-12$ & 100 \\
direct & $7.9168 \mathrm{e}-10$ & 1000 \\
revised & $1.7551 \mathrm{e}-5$ & 100 \\
revised & $1.7528 \mathrm{e}-7$ & 1000 \\
\hline
\end{tabular}

Example 5. $c(s)=s, \phi(s)=0, f(t)=1+2 t^{0.5}-t+t^{3} / 6, x(t)=t$.

\begin{tabular}{ccc}
\hline method & Max error & $n$ \\
\hline direct & $6.2783 \mathrm{e}-12$ & 100 \\
direct & $7.7382 \mathrm{e}-10$ & 1000 \\
revised & 0.0025 & 100 \\
revised & $5.3542 \mathrm{e}-5$ & 1000 \\
\hline
\end{tabular}


Example 6. $c(s)=s, \phi(s)=s^{2}, f(t)=-13 / 12+16 t / 3-t^{2} / 2, x(t)=t^{2}$.

\begin{tabular}{ccc}
\hline method & Max error & $n$ \\
\hline direct & 11.7022 & 100 \\
direct & 11.5983 & 1000 \\
revised & 0.0270 & 100 \\
revised & 0.0047 & 1000 \\
\hline
\end{tabular}

\section{Summary}

In this study, we present numerical methods for solving a class of hybrid integro-differential equations; the equation of the first kind originates from an aeroelasticity model. The direct method from previous study provides satisfactory results essentially for the linear cases. For the nonlinear cases, a revised method is proposed to obtain more accurate results.

\section{References}

[1] Burns, J.A., Cliff, E.M. and Herdman, T.L. (1983) A State-Space Model for an Aeroelastic System. 22nd IEEE Conference on Decision and Control, 1074-1077. https://doi.org/10.1109/CDC.1983.269685

[2] Burns, J.A., Herdman, T.L. and Stech, H.W. (1983) Linear Functional Differential Equations as Semigroups on Product Spaces. SIAM Journal on Mathematical Analysis, 14, 98-116. https://doi.org/10.1137/0514007

[3] Chen, H.-H. and Chiang, S. (2009) A Specific Procedure for Analytic Solutions to a Class of Singular Integral Equations. Chung Hua Journal of Computational Science, 7, 29-34.

[4] Chiang, S. (2005) Notes on the Solution of a Class of Singular Integral Equations. Chung Hua Journal of Science and Engineering, 3, 89-95.

[5] Chiang, S. (2006) On the Numerical Solution of a Class of Singular Integro-Differential Equations. Chung Hua Journal of Science and Engineering, 4, 43-48.

[6] Kappel, F. and Zhang, K.P. (1986) Equivalence of Functional Equations of Neutral Type and Abstract Cauchy Problems. Monatshefte für Mathematik, 101, 115-133. https://doi.org/10.1007/BF01298925

[7] Chiang, S. (2016) Numerical Algorithm and Its Revision for Solving a Class of Nonsingular Integro-Differential Equations of the First Kind. Chung Hua Journal of Computational Science, 6, 15-23. 
Submit or recommend next manuscript to SCIRP and we will provide best service for you:

Accepting pre-submission inquiries through Email, Facebook, LinkedIn, Twitter, etc. A wide selection of journals (inclusive of 9 subjects, more than 200 journals)

Providing 24-hour high-quality service

User-friendly online submission system

Fair and swift peer-review system

Efficient typesetting and proofreading procedure

Display of the result of downloads and visits, as well as the number of cited articles Maximum dissemination of your research work

Submit your manuscript at: http://papersubmission.scirp.org/

Or contact am@scirp.org 